\title{
Impact of beryllium surface layers on deuterium retention in tungsten
}

\author{
R. P. Doerner ${ }^{a^{*}}$, M. J. Baldwin ${ }^{a}$, D. Nishijima ${ }^{a}$, J. Roth ${ }^{b}$, and K. Schmid ${ }^{b}$ \\ ${ }^{a}$ Center for Energy Research, University of California at San Diego, La Jolla, CA 92093-0417, USA. \\ ${ }^{b}$ Max-Plank-Institut für Plasmaphysik, Garching, Germany.
}

\begin{abstract}
In ITER mixed Be/W layers are likely to form and their retention behavior is unknown. A series of ITER-grade tungsten samples have been exposed in PISCES-B to deuterium plasma at $300^{\circ} \mathrm{C}$, with and without beryllium impurity seeding of the plasma. If the beryllium concentration is small, the majority of the incident beryllium is re-eroded from the sample surface and only a few nm thick layer of mixed Be/W remains. The $\mathrm{W}$ samples exposed without beryllium seeding showed blister formation. The inclusion of $0.2 \%$ beryllium impurity ion fraction within the plasma eliminated the surface blisters, however did not change the amount of retained deuterium in the samples. When the beryllium impurity concentration in the plasma is large (2.5\%), a beryllium layer grows as the fluence increases. The deuterium retention also increases and exceeds the amount retained in either the pure tungsten, or the mixed Be-W layer on tungsten, case.
\end{abstract}

JNM Keywords: Plasma-materials interactions, Beryllium, Tungsten, Deuterium PSI Keywords: PISCES-B, Deuterium Inventory, Beryllium, Tungsten, Surface Analysis PACS: 52.40.Hf

Corresponding author address: UCSD, 9500 Gilman Dr. MC-0417, La Jolla, CA. 920930417, USA

Corresponding author e-mail: rdoerner@ucsd.edu

Presenting author: R. P. Doerner

Presenting author e-mail: rdoerner@ucsd.edu 


\section{Introduction}

The plasma-surface interactions in magnetically confined fusion devices are critical in several areas of a design team's consideration. One area is the impurity production rate, both the steady-state erosion rate and the material loss rate of the plasma-facing surface during transient events, will play major roles in determining the lifetime of the plasma-facing component. Another area where plasma-surface interactions can present a problem is the ability of the material to retain plasma fuel species. The amount of tritium retention in the plasma-facing materials will be governed by issues such as mobility within the material and recombination from the material surface. Ultimately, the accumulation rate of tritium within the confinement vessel will determine the operation period between machine shutdowns necessary to stay within prescribed safety limits.

In the ITER design activity, tradeoffs between these and other issues have resulted in the selection of three different plasma-facing material surfaces: namely beryllium (Be), carbon (C) and tungsten (W). A preliminary estimate of the tritium accumulation rate of the present ITER design has been carried out [1], however this estimate does not take into account such effects as transient surface heating events, neutron damage of materials, nor mixing of the three elements once plasma is initiated. The influence of beryllium mixing with the carbon has previously been reported [2, 3]. This paper examines the effect on retention of the mixing between beryllium impurity ions incident on a tungsten target plate. Deuterium retention in clean tungsten during both pure $\mathrm{D}$ and $\mathrm{D} / \mathrm{He}$ mixture plasma exposure has been investigated by several research groups [4-9].

Prior experiments in PISCES-B [10] have examined the alloy formation conditions for the $\mathrm{Be} / \mathrm{W}$ system [11]. While these experiments focused on the surface compositional changes under various plasma conditions, there was some evidence that Be in the W surface will have an impact on the deuterium retention in the samples. Retention is $\mathrm{W}$ is typically associated 
with the appearance of blisters on the surface of the material $[4,5]$. When Be is present in the incident plasma, it is observed to impede the formation of the blisters. A dramatic example of this behavior using a polycrystalline $\mathrm{W}$ sample is shown in Figure 1(a and b), although the retention in the samples shown here could not be quantified, due to technical difficulties, the retention was observed to drop due to the presence of the Be.

\section{Experimental procedure}

To quantify the change in retention a series of ITER-grade $\mathrm{W}$ samples (i.e. W samples conforming to ASTM B760 - Standard Specification for Tungsten Plate, Sheet, and Foil, where the grain orientation is perpendicular to the surface where the heat flux is applied) were exposed in PISCES-B [10] to deuterium plasma, with and without external seeding of the plasma with Be impurities. The amount of Be impurities can be independently controlled by changing the temperature of a Be effusion cell. An absolutely calibrated visible and nearUV spectrometer is used to measure Be line emission from the plasma column and the line emission intensity is converted into a Be density using photon emission coefficients obtained from the ADAS data base [12]. Plasma parameters are measured by a fast reciprocating double tipped Langmuir probe.

The $\mathrm{W}$ samples were mechanically polished and were pretreated before plasma exposure by annealing at $1000^{\circ} \mathrm{C}$ in vacuum for 15 minutes. It should be noted that due to the nature of the ITER-grade W material, with grains oriented preferentially normal to the surface, and the relatively thin $(1 \mathrm{~mm})$ samples used in PISCES-B, the force exerted on the center of the back surface of the sample by a thermocouple was enough to cause cracking of the plasmaexposed front surface. The nature of the sample cracking facilitated breaking the samples into two pieces following plasma exposure, so that separate retention measurements could be made on material exposed to the same plasma. 
Deuterium retention was measured in the plasma exposed samples in two ways. One piece of the target was shipped to IPP Garching for nuclear reaction analysis (NRA) to determine the areal density of both deuterium and beryllium. The NRA data provides a value for the elemental density averaged over the penetration depth of the incident ion beam, in this case about 3-4 microns. These samples were subsequently analyzed by SIMS depth profiling to determine the depth profile of the mixed Be/W surface layer. The other half of the samples were desorbed at UCSD using calibrated thermal desorption spectrometry (TDS) with a temperature ramp rate of $0.5 \mathrm{~K} / \mathrm{sec}$.

All the samples were exposed at $300^{\circ} \mathrm{C}$, where previous data have shown a peak in the deuterium retention in $\mathrm{W}$ [1]. Each sample was exposed to deuterium plasma flux of between 1 and $4 \times 10^{22} \mathrm{~m}^{-2} \mathrm{~s}^{-1}$, while adjusting the duration of the exposure to obtain the required fluence to each sample. An incident ion energy of $45-55 \mathrm{eV}$ was obtained by applying a negative bias to the samples.

\section{Experimental results}

As expected, the ITER-grade $\mathrm{W}$ targets exposed to deuterium-only plasma exhibited surface blistering as shown in Figure 2, although at a reduced amount compared to the polycrystalline $\mathrm{W}$ samples shown in Figure 1 . The deuterium release behavior from the samples, shown in Figure 3, is consistent with low fluence release results [6] where an increase in the release at $800 \mathrm{~K}$ was observed as the fluence increased. In our case the lower temperature release at 400-500 K [6] was not detected since the exposure temperature was above this value. There may be a slight shift with fluence in the release temperature to higher values, but the data could also result from a superposition of two release peaks and an increase in the amount being released at $800 \mathrm{~K}$. The ITER-grade samples exposed to the mixed D/Be plasma did not show any surface blister features. The deuterium release behavior 
from the $\mathrm{W}$ samples exposed to mixed D/Be plasma was significantly different than that measured from the samples exposed to pure D plasma. As shown in Figure 4, the release shows an additional release peak at higher temperature (around $1000 \mathrm{~K}$ ) with the presence of the mixed Be/W surface layer present.

The integrated retention for both sets of samples is plotted in Figure 5. For the case of the samples exposed without Be seeding the increase in retention is almost linear with fluence, while for the Be exposed $\mathrm{W}$ samples the retention appears to exhibit a saturation with increasing fluence, but additional high fluence exposures will be needed to verify the saturation behavior. Also shown in this figure is the areal density measured within the first 3 microns of the surface as measured by NRA. In the case of the samples exposed to pure D plasma, the NRA measurement shows a constant value with increasing fluence. This indicates that retention within the first 3 microns has already saturated during the lowest fluence exposure. The increased retention with fluence measured by TDS must be coming from depths not accessible to the NRA measurement. In the case of the samples exposed to mixed $\mathrm{D} /$ Be plasma the areal density measured by NRA is considerably less and does not show any consistent change with increasing fluence. This indicates that while the Be/W surface layer inhibits the formation of blisters and reduces the retention within the first 3 microns, it does not alter the global retention within the $\mathrm{W}$ bulk.

SIMS analysis of the samples was used to determine the thickness of the mixed Be/W surface layer. The SIMS analysis was performed using a $2 \mathrm{keV} \mathrm{Ar}$ ion beam. The beam was scanned over a $1 \mathrm{x} 1 \mathrm{~mm}^{2}$ area with a beam current of $\sim 70 \mathrm{nA}$. The depth profiles of Be are shown in Figure 6. The depth scale was calculated assuming the sputtering yield of Be by 2 $\mathrm{keV} \operatorname{Ar}$ ions ( 0.9 atoms/ion) [13]. While the medium fluence sample appears to have a thicker Be surface layer than the highest fluence sample, all the Be layers are on the order of only a few nm thick. 
One final W sample was exposed to Be-containing plasma to demonstrate the importance of the plasma conditions on the retention in plasma-exposed surfaces. In this case, the incident plasma flux was reduced while keeping the seeding rate of Be constant. The result was a situation where a Be layer continuously deposited on the $\mathrm{W}$ surface during the exposure. Based on new calculations of the BeII photon emission coefficient [12] and the absolutely calibrated spectroscopy system that is now capable of measuring BeII resonant transition at $313.1 \mathrm{~nm}$, it appears that previous measurements [14] using the $467.3 \mathrm{~nm}$ emission in PISCES experiments have been underestimating the concentration of Be impurities in the plasma. The new measurements and calculations increase the Be fraction in the plasma by roughly a factor of 8 over previous measurements. A simple estimate of the particle balance for this experiment gives some confidence in these new Be calculations.

For this experiment, the Be fraction in the plasma is estimated to be $2.5 \%$. Previous measurements of the sputtering yield for plasma deposited Be layers [15] at this ion energy (55 eV) and surface temperature (573K) give a value of 4e-3. Assuming that the reflection coefficient of Be from W is similar to that calculated for C from W [13] it should be close to 50\%. The net Be deposition on the surface should therefore be,

$$
((0.025 / 2)-0.004) \times \Gamma_{\text {in }} \sim 0.0085 * 3.5 \times 10^{21} \mathrm{~m}^{-2} \mathrm{~s}^{-1} \sim 3 \times 10^{19} \mathrm{~m}^{-2} \mathrm{~s}^{-1}
$$

and for the $2000 \mathrm{sec}$ duration of the experiment one should expect $6 \times 10^{22} \mathrm{~m}^{-2}$. This agrees well with the amount of Be measured on the surface using NRA of $6 \times 10^{22} \mathrm{~m}^{-2}$. And the measured D/Be value of 0.03 agrees with the value predicted for Be rich codeposited/ coimplanted surfaces at these formation conditions of 0.036 [17].

In addition to verifying a more accurate measurement of the Be fraction in the plasma, this sample also provided the retention of $\mathrm{D}$ in the growing Be layer. The total $\mathrm{D}$ retained in the layer measured by NRA was $3 \times 10^{17} \mathrm{~cm}^{-2}$. This level of D retention is more than an order of magnitude higher than those measured when the $\mathrm{Be}$ is in a net erosion regime, as was 
shown in Figure 5.

\section{Discussion and Conclusion}

These retention measurements show that the impact of a thin Be layer on $\mathrm{W}$ resulting during an erosion dominated exposure is relatively small. Similar results are found when comparing $\mathrm{D}+\mathrm{He}+\mathrm{Be}$ plasma exposure of $\mathrm{W}$ to pure $\mathrm{D}$ plasma exposure of $\mathrm{W}$ [16]. While the global value of retention is unaffected within the present fluence range by the thin Be surface layer, the details may provide insight into the fundamental mechanisms of retention in $\mathrm{W}$.

It has been seen that retention in $\mathrm{W}$ tends to correlate to the existence of surface blistering $[4,5]$. This is also observed during these pure D plasma exposures, where the amount of D in the first few microns of the surface agrees with the global retention until one reaches very large fluence. However, these measurements show that surface Be tends to eliminate blister formation. This can be seen by SEM observation of the sample surface, as well as by the reduced amount of $\mathrm{D}$ retained in the first few microns of the surface during the Be seeded experiments.

There have been several possible mechanisms suggested for ways that Be could inhibit surface blisters. First, a small amount of Be may be migrating along grain boundaries present in the $\mathrm{W}$ surface. This small amount of Be appearing in the grain boundaries may prevent the formation and/or growth of cavities in these regions that would trap deuterium and lead to a pressure increase eventually delaminating the grain, or region above the cavity, causing a surface blister. It has also been speculated that the presence of a Be layer in the implantation region of the incident $\mathrm{D}$ ions could act to reduce the stress field resulting from a super saturation of D in this region. Without the stress field, the driving force for blister formation deeper in the material might not exist. Whether either of these explanations is correct cannot be determined from the data presented here and will require further detailed experiments. 
However, it is interesting to note that in these experiments, the global amount of retention observed in either set of samples did not change appreciably with or without blisters on the surface of the $\mathrm{W}$. This indicates that while blisters may be responsible for accumulating $\mathrm{D}$ in the bulk of $\mathrm{W}$ samples when they occur, there are other trapping sites within the $\mathrm{W}$ that can still fill with $\mathrm{D}$ once the migrating $\mathrm{D}$ proceeds beyond the range of the blister cavities. In any case, whether a thin Be surface layer exist or doesn't, the retention in W does not appear to be large compared to the retention in depositing material. Of course, other issues affecting retention such as neutron damage and transient events will still need to be evaluated.

These measurements show that D retention increases dramatically only when the plasma condition changes from an erosion dominated regime to a deposition dominated regime. When the Be layer thickness begins to grow with time, the amount of $\mathrm{D}$ retained in the growing Be layer will become much larger than the amount retained in the $\mathrm{W}$ bulk. Depending on the deposition conditions, such layers could become quite thick and would need to be accounted for during tritium accumulation estimates in ITER.

\section{Acknowledgements}

Work supported by DOE grant DE-FG02-07ER54912 and the EU PWI Task Force.

\section{References}

[1] J. Roth et al., Plasma Phys. Control. Fusion 50 (2008) 103001.

[2] R. P. Doerner et al., J. Nucl. Mater. 266-269 (1999) 392.

[3] M. J. Baldwin et al., J. Nucl. Mater. 358 (2006) 96.

[4] M. Miyamoto et al., Nucl. Fusion 49(2009)065035.

[5] V. Kh Alimov et al., Phys. Scripta T138 (2009) 014048.

[6] O.V. Ogorodnikova et al., J. Appl. Phys. 103 (2008)034902. 
[7] G. M. Wright et al., Nucl. Fusion 50 (2010)055004.

[8] K. Tokunaga et al., J. Nucl. Mater. 337-339 (2005)887.

[9] W. R. Wampler et al., Nucl. Fusion 49 (2009)115023.

[10] R. P. Doerner et al., Phys. Scripta T111(2004)75.

[11] M. J. Baldwin et al., J. Nucl. Mater. 363-365 (2007)1179.

[12] H. P. Summers, Atomic Data and Analysis Structure - User Manual, Rep. JET-IR(94)

(JET Joint Undertaking, Abingdon, 1994).

[13] W. Eckstein, Calculated sputtering, reflection and range values, IPP Report 9/132, June 2002.

[14] D. Nishijima et al., J. Nucl. Mater. 363-365 (2007) 1261.

[15] D. Nishijima et al., J. Nucl. Mater. 390-391 (2009) 132.

[16] M Miyamoto et al., these procedings. 

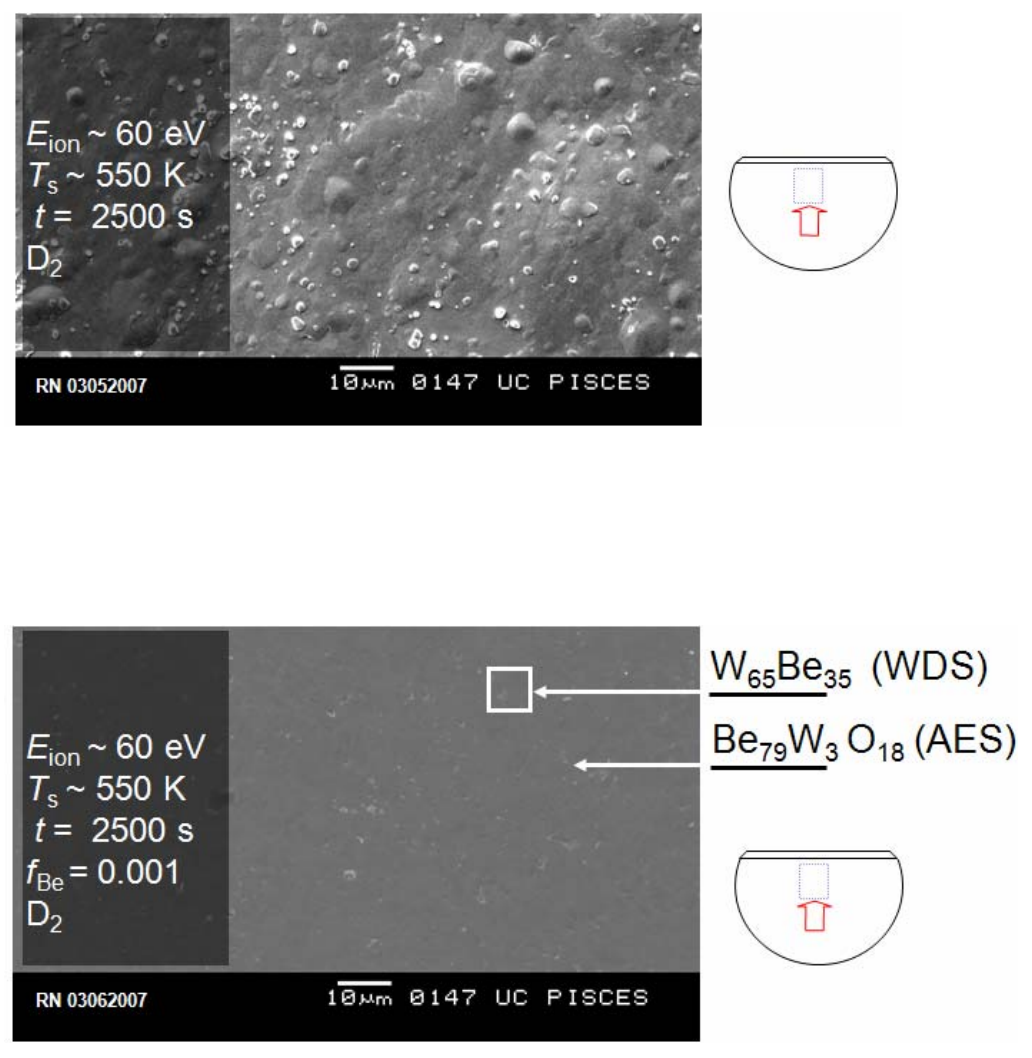

Figure 1 - Surface of polycrystalline tungsten samples exposed to D plasma (above) and Be containing D plasma (below). 


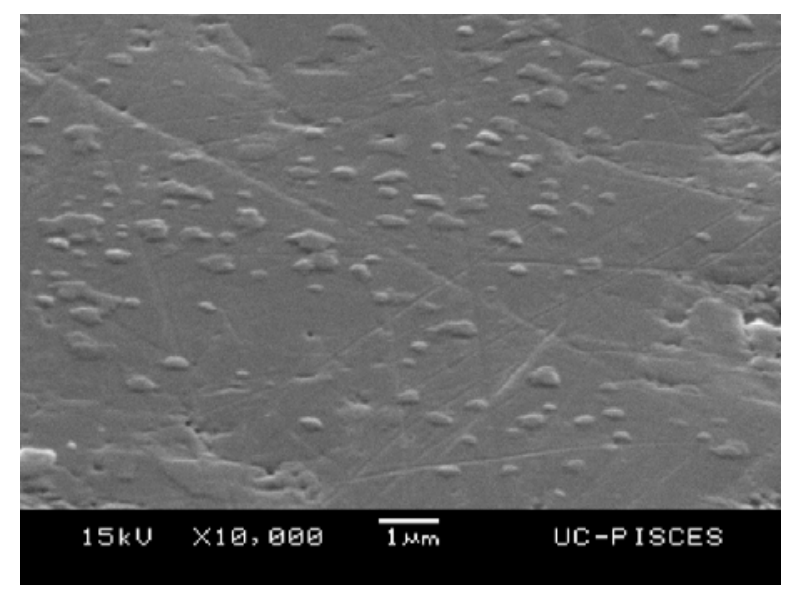

Figure 2 - Surface of ITER-grade tungsten sample after exposure to D plasma showing the formation of small surface blisters. 


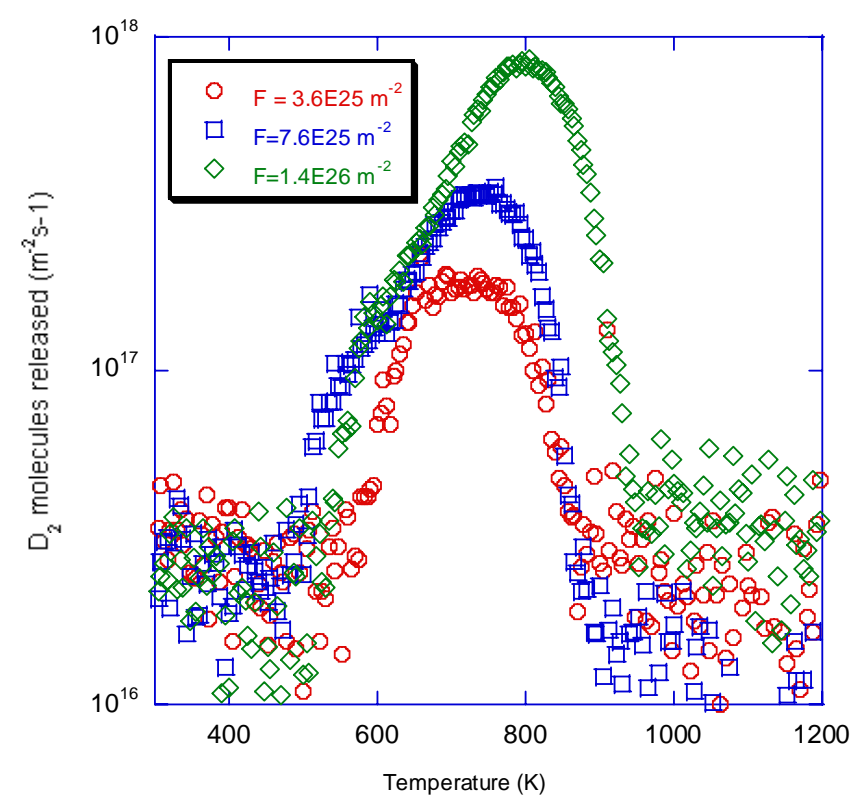

Figure 3 - Deuterium release from tungsten exposed to D plasma. 


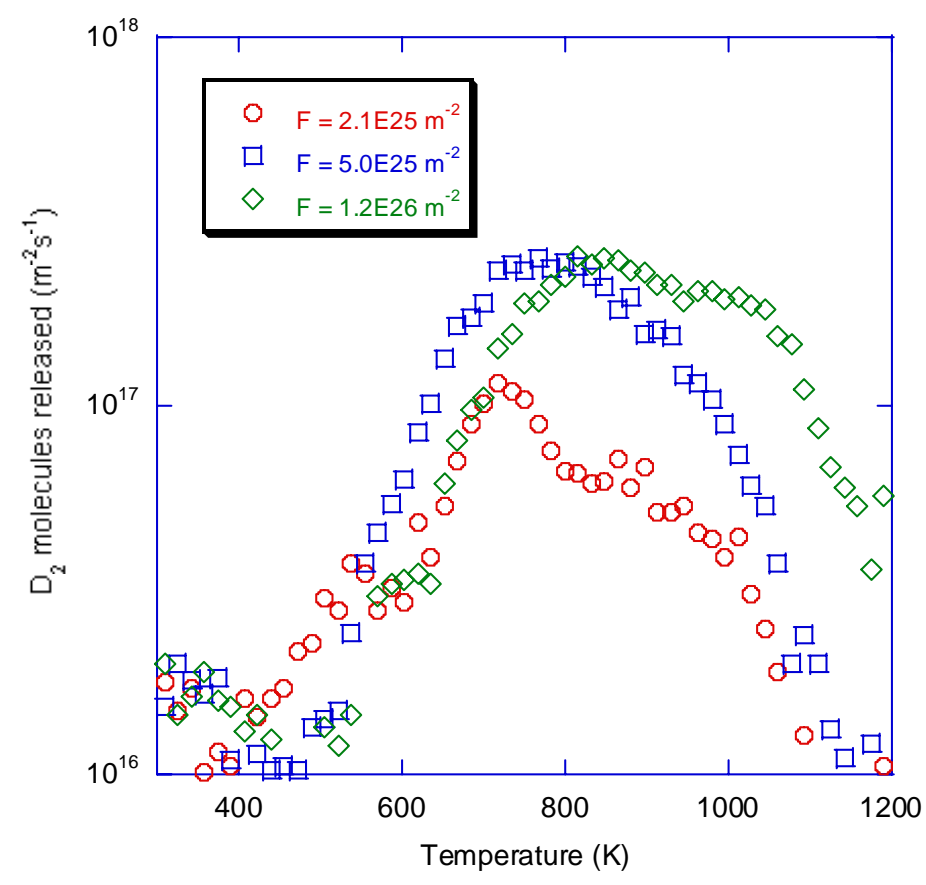

Figure 4 - Deuterium release from tungsten exposed to Be-containing D plasma. 


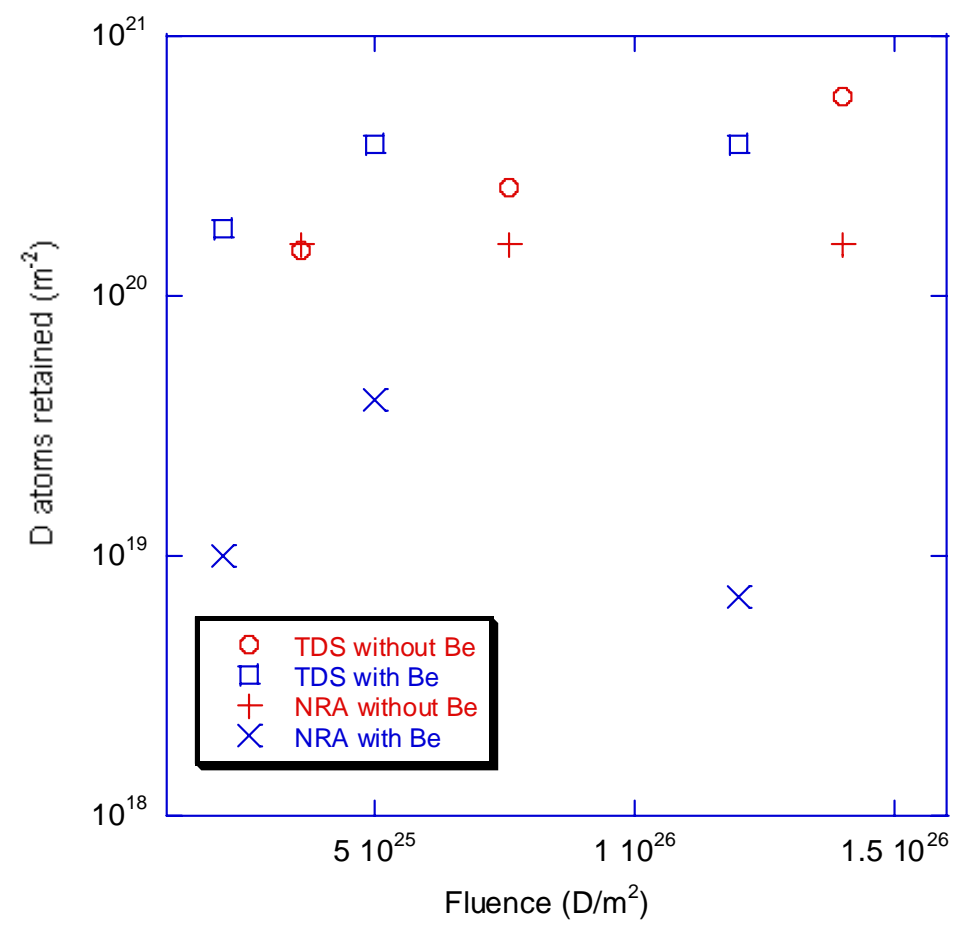

Figure 5 - Total retention measured using TDS and NRA from tungsten samples exposed to $\mathrm{D}$, and Be-containing $\mathrm{D}$, plasma. 


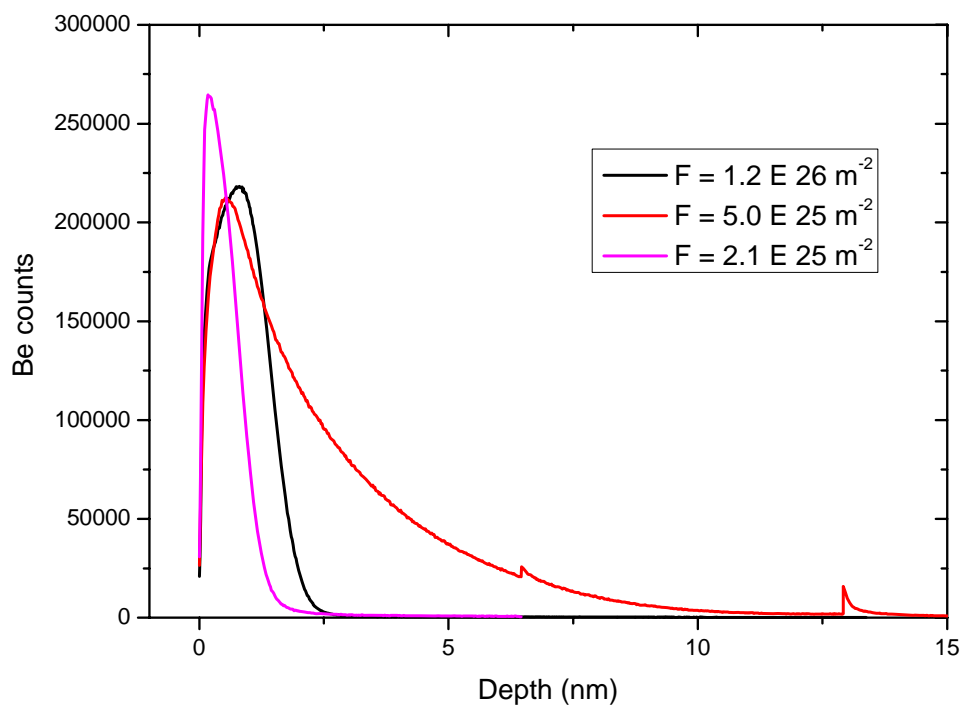

Figure 6 - SIMS depth profiles of Be/W mixed layer after Be-containing D plasma exposure. 